\title{
HD 150136: towards one of the most massive systems?
}

\author{
Laurent Mahy ${ }^{1}$, Eric Gosset ${ }^{1}$, Hugues Sana ${ }^{2}$, Gregor Rauw ${ }^{1}$, \\ Thomas Fauchez ${ }^{1}$ and Christian Nitschelm ${ }^{3}$ \\ ${ }^{1}$ Institut d'Astrophysique et de Géophysique, University of Liège, \\ Allée du 6 Août, 17, Bât. B5C, B-4000, Liège, Belgium \\ email: mahy@astro.ulg.ac.be \\ ${ }^{2}$ Sterrenkundig Instituut 'Anton Pannekoek', Universiteit van Amsterdam, \\ Postbus 94249, NL-1090 GE Amsterdam, The Netherlands \\ ${ }^{3}$ Instituto de Astronomía, Universidad Católica del Norte, \\ Avenida Angamos 0610, Antofagasta, Chile
}

\begin{abstract}
We present the preliminary results of an intensive monitoring devoted to HD 150136 . Already quoted as an $\mathrm{O} 3+\mathrm{O} 6$ binary, we detected a third O-type component physically linked to the system, making it one of the nearest $(1.3 \mathrm{kpc})$ most massive systems known until now $\left(\sim 134 M_{\odot}\right)$. To determine the physical parameters of this system, we applied a disentangling program to study individually the three components. It allows us to constrain their spectral types and to derive a new orbital solution for the short-period system.
\end{abstract}

Keywords. stars: individual (HD 150136), binaries: spectroscopic, stars: fundamental parameters

\section{Overview on HD 150136}

HD 150136 was classified by Niemela \& Gamen (2005) as an O3V+O6V binary system. This system has an orbital period of about 2.66 days and presents variability in the X-ray domain on a one-day time scale (Skinner et al. 2005).

Sixty-four spectra of HD 150136 were collected from 1999 to 2006 and 14 others in 2009 with the $1.5 \mathrm{~m}$ and $2.2 \mathrm{~m}$ telescopes, at La Silla, equipped with FEROS. These highresolution spectra allowed us to detect a third component and to derive spectral types of O3, O6 and O6.5-O7 for the primary, the secondary and the third star, respectively.

\section{Orbital solution of the short-term binary and properties of the third star}

We used a disentangling program, based on the method of González \& Levato (2006) and adapted to triple systems, which also measures the radial velocities (RVs) by crosscorrelation even at phases where the spectra are heavily blended. We applied a Fourier method (Heck et al. 1985) to the differences of these RVs to refine the orbital period of the short-term system. This yields a period of $2.67 \pm 0.01$ days, i.e., similar to the previous one (Niemela \& Gamen 2005). The RV curve is given in Fig. 1 (left panel) whilst the orbital parameters are listed in Table 1 ( $T_{0}$ refers the time of the primary conjunction). We fitted the primary and the secondary by using the CMFGEN atmosphere code (Hillier \& Miller 1998). The stellar parameters were constrained as in Mahy et al. (2010) but we were not able to estimate the wind parameters because the wind diagnostic lines, in the optical domain, present variations impossible to disentangle. We derived $T_{\text {eff }}$ of about 

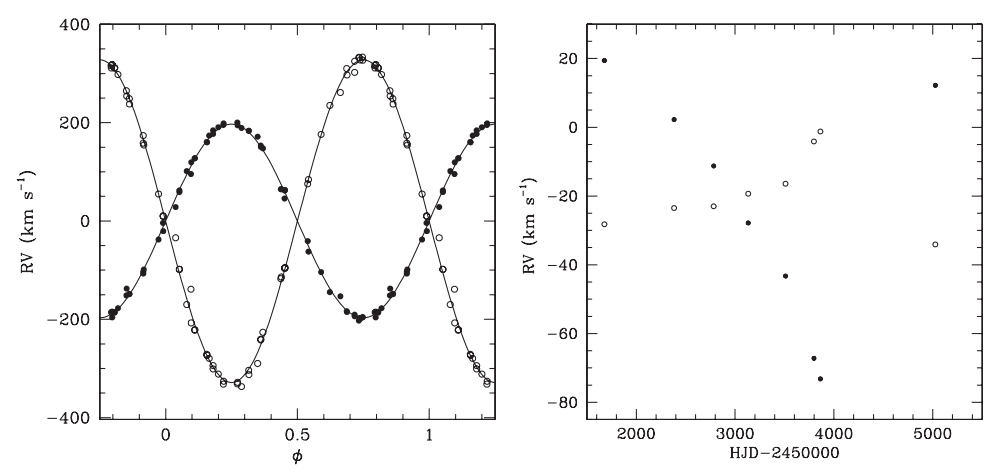

Figure 1. Left: RV curve of the short-term binary system. Full circles indicate the primary, the open ones represent the secondary star. Right: Evolution of the RVs of the third component and the mean systemic velocity of the short-period binary (full/open circles) as a function of time.

Table 1. Orbital parameters of the short-term binary system.

\begin{tabular}{lcc}
\hline Parameters & Primary & Secondary \\
\hline$P($ days $)$ & \multicolumn{2}{c}{$2.67 \pm 0.01$} \\
$e$ & \multicolumn{2}{c}{0.0 (fixed) } \\
$T_{0}(\mathrm{HJD})$ & $2451318.518 \pm 0.002$ \\
$K\left(\mathrm{~km} \mathrm{~s}^{-1}\right)$ & $196.9 \pm 1.0$ & $328.3 \pm 1.7$ \\
$a \sin i\left(R_{\odot}\right)$ & $10.4 \pm 0.1$ & $17.3 \pm 0.1$ \\
$M \sin ^{3} i\left(M_{\odot}\right)$ & $25.1 \pm 0.3$ & $15.0 \pm 0.2$ \\
$Q\left(M_{1} / M_{2}\right)$ & $1.667 \pm 0.009$ \\
$\mathrm{rms}\left(\mathrm{km} \mathrm{s}^{-1}\right)$ & \multicolumn{2}{c}{9.75} \\
\hline
\end{tabular}

45.7 and $39.8 \mathrm{kK}, \log \left(L / L_{\odot}\right)$ of 5.85 and 5.43 and $\log g$ of 4.0 , for the primary and the secondary, respectively, suggesting respective masses of 67 and $40 \mathrm{M}_{\odot}$.

The evolution with time of the RVs of the third component and of the mean systemic velocity of the short-period binary (Fig. 1, right panel) reveals, for the first time, that the three components are physically linked. The expected period of this long-term system is certainly larger than 10 years and the orbit is clearly eccentric.

\section{Future works and conclusions}

The high-resolution of our data allowed us to show the existence of a 3rd component in the HD 150136 system. However, we are not yet able to constrain with high accuracy the parameters of this 3rd star. The short-term binary system is composed of O3V and O6V stars, probably in contact (Skinner et al. 2005), with an inclination close to $46^{\circ}$. This system also likely features a wind interaction zone, as revealed by the complex profile variations of the He II 4686 and $\mathrm{H}_{\alpha}$ lines. This will be investigated in a future paper.

\section{References}

González, J. F. \& Levato, H. 2006, A\&GA, 448, 283

Heck, A., Manfroid, J., \& Mersch, G. 1985, A 8 AS, 59, 63

Hillier, D. J. \& Miller, D. L. 1998, ApJ, 496, 407

Mahy, L., Rauw, G., Martins, F., Nazé, Y. et al. 2010, ApJ, 708, 1537 ApJ, 708, 1537

Niemela, V. S. \& Gamen, R. C. 2005, MNRAS, 356, 974

Skinner, S. L., Zhekov, S. A., Palla, F., \& Barbosa, C. L. D. R. 2005, MNRAS, 361, 191 